\title{
Reposicionamiento del Barrio del Artista para aumentar su valor cultural-artístico en Puebla, México
}

\section{Repositioning of the Barrio del Artista to increase its cultural-artistic value in Puebla, Mexico}

Ramón Sebastián Acle-Mena'
Carolina Villalobos-Abrego $^{2}$
Beatriz Herrera-López $^{3}$

Recibido: junio 02 de 2014 Aceptado: agosto 28 de 2015

\begin{abstract}
Resumen
El objetivo general del presente trabajo fue determinar la correlación del reposicionamiento del Barrio del Artista para aumentar su valor cultural-artístico en Puebla, México. La investigación se realizó dentro de un enfoque mercadológico. Los resultados que se presentan corresponden a un estudio empírico sobre el reposicionamiento del Barrio del Artista, usando un enfoque cuantitativo y el coeficiente de correlación de Pearson, logrando la aplicación de los factores que componen el posicionamiento para determinar la relación entre el reposicionamiento del Barrio del Artista y el aumento de su valor cultural artístico en Puebla. Para la presente investigación se utilizaron de forma práctica los conceptos novedosos de mercadotecnia y posicionamiento.
\end{abstract}

Palabras clave: mercadotecnia, posicionamiento, Barrio del Artista.

\begin{abstract}
The main aim of the current study was to identify the correlation of the Barrio del Artista repositioning to enhance its artistic and cultural value in Puebla city, Mexico. The research was conducted within a marketing focus. The presented results correspond to an empirical research on the repositioning of the Barrio del Artista, using a quantitative approach and Pearson's correlation coefficient, making the application of the factors that compose positioning to determine the relationship between the Barrio del Artista repositioning and the increase of its artistic and cultural value in Puebla city. For this research, the novel concepts of marketing and positioning were used in a practical way.
\end{abstract}

Keywords: marketing, positioning, Barrio del Artista.

\footnotetext{
1 Doctor en Administración Gerencial, Benemérita Universidad Autónoma de Puebla, México. E-mail: raclemx@hotmail.com

2 Licenciada en Turismo, Benemérita Universidad Autónoma de Puebla, México. E-mail: morcykarolin@hotmail.com

3 Licenciada en Turismo, Maestra en Gestión de Patrimonio Natural y Cultural, Benemérita Universidad Autónoma de Puebla, México.

E-mail: beatrizherreralopez@gmail.com
} 


\section{Introducción}

La investigación trata sobre el reposicionamiento para aumentar el valor cultural-artístico del Barrio del Artista en la ciudad de Puebla, México. La problemática principal surge de la falta de estudios teóricos y de campo que reflejen la relación entre el reposicionamiento del Barrio del Artista para aumentar su valor cultural artístico en Puebla, dentro del contexto mercadológico. Por lo anterior surge el interrogante de ¿qué es necesario para resolver esta problemática? La respuesta está en reposicionar el Barrio del Artista para aumentar su valor artístico cultural.

La importancia de la investigación radica en que es un trabajo empírico, original y específico que aporta beneficios teóricos y metodológicos. Este trabajo se considera relevante al no existir muchos estudios a cerca de las problemáticas del Barrio del Artista si se refiere a su valor cultural y artístico.

El objetivo general fue determinar la correlación del reposicionamiento del Barrio del Artista para aumentar su valor cultural artístico en Puebla, con la finalidad de direccionar el estudio. De esta forma, se planteó la siguiente hipótesis de tipo correlacional: a través del reposicionamiento del Barrio del Artista se aumentará su valor cultural artístico en Puebla; en cuanto a la operacionalización de las variables, se considera como variable independiente el reposicionamiento del Barrio del Artista, siendo la variable dependiente el aumento de su valor cultural artístico en Puebla.

El presente documento contiene un marco teórico, donde se realizó una revisión de la literatura para tener claridad sobre los conceptos de mercadotecnia, posicionamiento y el Barrio del Artista. Luego se presenta la metodología de la investigación, los resultados y conclusiones. Se destaca que existen varios factores determinantes de posicionamiento para aumentar el valor cultural y artístico del Barrio del Artista; estos factores pueden afectar en forma directa o indirecta a los turistas nacionales e internacionales, así como a los visitantes locales para su visita a este espacio.

\section{Materiales y métodos}

\subsection{Marco teórico}

Se considera que la mercadotecnia es una herramienta indispensable para toda empresa que busque reconocer necesidades, deseos, innovar, distribuir, fijar precios y promocionar bienes y servicios, asegurando así su permanencia en el mercado. Los principales objetivos de la mayoría de las empresan son sobrevivir, obtener utilidades y crecer. La mercadotecnia contribuye directamente al logro de estos objetivos e incluye las siguientes actividades que son vitales para las organizaciones de negocios: evaluar los deseos y satisfacciones de los clientes actuales y potenciales, diseñar y manejar ofertas de productos, determinar precios y políticas de fijación de precios, desarrollar estrategias de distribución y comunicarse con clientes actuales y potenciales (Acle, Villalobos, Saniesteban, Rodríguez, \& Zamora, 2014).

Las actividades de mercadotecnia contribuyen en forma directa a la venta de los productos y servicios en una organización, permitiendo satisfacer de manera más completa las cambiantes necesidades de los consumidores. De esta manera, se ayuda a generar mayores utilidades en la empresa, lo que ayuda no solo a la supervivencia de los negocios particulares, sino también al bienestar de la economía en general. La mercadotecnia está en plena evolución, siendo una nueva corriente que ayuda a las organizaciones a sobrevivir en el mercado actual (Fischer \& Espejo, 2007).

Kotler y Amstrong (2001, p. 3) definen la mercadotecnia como "un proceso social y administrativo mediante el cual los individuos y los grupos obtienen lo que necesitan y desean, creando e intercambiando valor con otros". Partiendo de esta definición se puede señalar que dentro de la función de la mercadotecnia está implícita la administración de la mezcla de mercadotecnia, 
que comprende el conjunto de variables controlables, identificadas quizá através del modelo mas conocido y aceptado en las escuelas de negocios, llamadas las cuatro P's: producto, precio, plaza y promoción. Este término se refiere a un diseño y combinación integral de estas 4 variables con el fin de producir intercambios mutuamente satisfactorios con un mercado-meta y lograr un éxito competitivo (Lamb, 2011).

En cuanto al posicionamiento, se le considera como una estrategia que permite a las empresas distinguirse de la competencia a través de ciertas características o atributos, logrando así ocupar los primeros sitios en las mentes de los clientes (Pride, 1997), obteniendo así una mayor competitividad y utilidad en el mercado. Las mercancías, servicios, compañías, instituciones e incluso personas son sujetas a ser posicionadas en la mente de los consumidores.

Este término se refiere a un sistema organizado que permite encontrar ventanas en la mente; dichos sistema se basan en la idea de que la comunicación solo puede tener lugar en el momento preciso y en las circunstancias apropiadas (Trout \& Rivkin, 1996). Consiste en lograr que un producto ocupe un lugar claro, distintivo y deseable en la mente de los consumidores-meta en relación con los productos de la competencia. Los consumidores definen un producto a partir de sus atributos importantes; es decir, el lugar que ocupa el producto en la mente de los clientes en relación con los productos de la competencia (Acle-Mena, Domínguez-Y-López, \& Biguete-García, 2013).

Una "posición" es la forma en que clientes actuales y posibles ven un producto, marca u organización en relación con la competencia (Mármol-Sinclair \& Ojeda-García, 2012). De igual manera, es el uso que hace una empresa de todos los elementos de que dispone para crear y mantener en la mente del mercado-meta una imagen particular en relación con los productos de la competencia (Stanton, Etzel, \& Walker, 2003). Posicionarse consiste simplemente en lograr que en una idea, o incluso en una palabra, que defina a la compañía esté en las mentes de los consumidores. Una fuerte identificación de marca representa una enorme ventaja para una compañía.

Actualmente, los consumidores están saturados con información sobre bienes y servicios, de manera tal que no pueden reevaluar los productos cada vez que toman la decisión de compra. Para facilitar la decisión de compra, los consumidores clasifican los productos en categorías; en otras palabras "posicionan" los productos, los servicios y las empresas dentro de un lugar en su mente. La posición de un producto depende de diversos factores como percepciones, impresiones y sentimientos que tienen los consumidores en cuanto al producto y en comparación con los productos de la competencia (Mármol-Sinclari \& Ojeda-García, 2012 ).

Como modelo de referencia para determinar las variables independientes en la presente investigación, se tomaron los tipos de posicionamiento propuestos por Lamb, Hair y Mc Daniel (2008):

Posicionamiento por atributo. Es cuando un producto se asocia con un atributo, característica del producto, o beneficio para el consumidor.

Posicionamiento por precio y calidad. Esta base de posicionamiento quizá haga hincapié en el precio alto como señal de calidad o destaque el precio bajo como advertencia de valor.

Posicionamiento por uso o aplicación. Resaltar usos o aplicaciones puede ser un medio efectivo para posicionar un producto con los compradores.

Posicionamiento por usuario del producto. Esta base de posicionamiento se dirige a un tipo o personalidad de usuario.

Posicionamiento por clase de producto. En este caso, el objetivo consiste en colocar el producto 
en asociación con una categoría particular de productos.

Posicionamiento por competidor. El posicionamiento contra los competidores es parte de cualquier estrategia de este tipo.

Posicionamiento por emoción. El posicionamiento que usa la emoción se enfoca en cómo el producto hace sentir a los clientes. Varias compañías usan este enfoque.

A nivel mundial, el posicionamiento ha llegado a ser el elemento más importante para la publicidad y la mercadotecnia. Este concepto ha revolucionado la comunicación publicitaria y la mercadotecnia. En nuestra sociedad sobre comunicada, el nombre del juego es posicionamiento. Es la estrategia competitiva que persigue "la excelencia y el éxito"; la que ayuda a determinar lo que se quiere hacer y cómo lograrlo exitosamente (Gambini, 2010).

Es necesario mencionar el contexto donde se desarrolló la investigación, que corresponde al sitio llamado Barrio del Artista, ubicado desde su fundación en la calle 8 norte y 6 oriente de la ciudad de Puebla, México. Es un espacio de difusión del arte fundado en el año de 1941 por José y Ángel Márquez Figueroa. Por décadas, la Unión de Artes Plásticas ha promovido la expresión de nuestras tradiciones dando oportunidad de un espacio a escuelas de bailes folklóricos y teatrales, organizando festivales en la plazuela y diversas festividades de carácter popular como: la quema de Judas, el rosario de la Virgen de Dolores, las ofrendas de Todos los Santos, la edición anual de las satíricas "Calaveras de mi Barrio", el festejar posadas y pastorelas en la época navideña y el arraigado jueves de Corpus Christi, festividad profano religiosa de nuestra cultura; así como la galería José Luis Rodríguez Alconedo, foro donde se muestra la actualidad de la música, la poesía y todo tipo de inquietudes culturales a diferentes niveles, contando con exposiciones individuales, colectivas y de invitados de todo el estado y del interior del país (Deana, 1986).

El Barrio del Artista ha sido fuente e inspiración de músicos y poetas que, amparados por el ambiente bohemio del lugar, han expresado su música y su poesía a través de diferentes épocas. Las visitas y convivencias de personajes de la talla de Diego Rivera y su esposa la señora Frida Kahlo, las amenas reuniones, para enjuagarse la boca, con el maestro Fernando Murillo, Doctor Atl., la presencia del Maestro David Siqueiros, del gran pintor Francisco Cornejo y de tantos otros intelectuales y estrellas del cine nacional que apoyaron el sueño del Barrio del Artista, que es y seguirá siendo un rincón en el Arte. Sin embargo, en los últimos años este espacio ha sufrido de un decrecimiento en cuanto al número de visitas por parte de los turistas locales y nacionales, que prefieren asistir a otro tipo de lugares durante su corta estancia en Puebla (Acle et al., 2014).

\subsection{Metodología}

De acuerdo con el marco teórico, se tomaron los factores que determinan el reposicionamiento y que conforman las variables independientes del estudio; esos factores son: atributo, precio y calidad, uso o aplicación, usuario del producto, clase de producto, competidor y emoción.

En la investigación se plantearon las siguientes hipótesis de trabajo, que sirvieron como guía para orientar el desarrollo del estudio, definiéndose los términos y variables, principalmente para que el investigador y los usuarios del estudio den el mismo significado a las variables y que permita certificar que puedan ser medidas, evaluadas e inducidas.

Hipótesis de trabajo:

H1: El atributo: fundamentado a través de: la arquitectura colonial y el ambiente bohemio, es un factor directamente proporcional al aumento del 
valor cultural artístico del Barrio del Artista en Puebla.

H2: El precio y calidad: fundamentados a través de: pinturas costosas; arte y cultura a costos elevados, es un factor directamente proporcional al aumento del valor cultural artístico del Barrio del Artista en Puebla.

H3: El uso o aplicación: fundamentados a través de: cursos o talleres y tradiciones y festividades populares, es un factor directamente proporcional al aumento del valor cultural artístico del Barrio del Artista en Puebla.

H4: El usuario del producto: fundamentado a través de: segmento cultural y conocimiento de arte y cultura, es un factor directamente proporcional al aumento del valor cultural artístico del Barrio del Artista en Puebla.

H5: La clase de producto: fundamentado a través de: lugar donde predomina el arte y la cultura; artistas pintando en sus talleres, es un factor directamente proporcional al aumento del valor cultural artístico del Barrio del Artista en Puebla.

H6: El competidor: fundamentado a través de: lugar único en México y no hay lugar que se le asemeje en Puebla, es un factor directamente proporcional al aumento del valor cultural artístico del Barrio del Artista en Puebla.

H7: La emoción: fundamentada a través de: sentirse relajado y lugar que vale la pena visitar, es un factor directamente proporcional al aumento del valor cultural artístico del Barrio del Artista en Puebla.
El diseño de la investigación consistió en un enfoque cuantitativo, utilizando la investigación exploratoria y correlacional, apoyándose en los métodos deductivo, analítico y sintético. En cuanto a la técnica de investigación se utilizó la encuesta y entrevista, aplicando un cuestionario que contenía 14 ítems y opciones de respuesta de escala tipo "Likert".

Las entrevistas se realizaron a 265 visitantes locales y turistas nacionales e internacionales, durante el período vacacional de Diciembre del 2013 en el Barrio del Artista. Por último se menciona el uso de las técnicas estadísticas coeficiente de correlación de Pearson y Alpha de Cronbach, apoyándose en los programas estadísticos de SPSS y Excel para correr y almacenar los datos recopilados.

Para determinar la correlación entre las variables independientes y la variable dependiente se aplicó la prueba paramétrica del coeficiente de correlación de Pearson (r). El coeficiente de correlación de Pearson es una prueba utilizada para analizar la relación entre dos variables medidas a un nivel intercalar o de razón. Se trata de un coeficiente de correlación paramétrico que indica con la mayor precisión cuándo dos cosas están correlacionadas; dicho de otra forma, hasta qué punto una variación en una cosa corresponde con una variación en otra. Sus valores varían de $+1,00$ que significa: correlación positiva perfecta; a través del cero significa independencia completa o ausencia de correlación, y hasta -1,00 significa una correlación perfecta negativa. La correlación perfecta de $+1,00$ está indicando que cuando una variable se mueve en una dirección, la otra se mueve en la misma dirección y con la misma intensidad. En la tabla 1 se presentan los significados de la correlación. 


\begin{tabular}{|c|c|}
\hline $\begin{array}{c}\text { Valor del coeficiente de correlación de } \\
\text { Pearson }\end{array}$ & Significado \\
\hline$r<0,20$ & Correlación leve, casi insignificante \\
\hline$r$ de 0,20 a 0,40 & Baja correlación, definida, pero baja \\
\hline$r$ de 0,40 a 0,70 & Correlación moderada, sustancial \\
\hline r de 0,70 a 0,90 & Correlación marcada, alta \\
\hline$r$ de 0,90 a 1,00 & Correlación altísima, muy significativa. \\
\hline
\end{tabular}

Tabla 1. Tabla de significados del coeficiente de correlación de Pearson (Acle-Mena \& Burguete-García, 2011).

\section{Resultados y discusión}

Las hipótesis, como proposiciones tentativas acerca de las relaciones entre dos o más variables, se someten a prueba en el campo por medio de una investigación diseñada especialmente para recolectar la información con los instrumentos de medición apropiados; del análisis e interpretación de los datos, se puede argumentar si las hipótesis son o no apoyadas. Los resultados de la investigación se resumen en la tabla 2, que sirven como base para probar o rechazar las hipótesis que se plantearon en el inicio del estudio sobre el reposicionamiento del Barrio del Artista para aumentar su valor cultural artístico en Puebla.

\begin{tabular}{ll}
\hline \multicolumn{1}{c}{ Hipótesis } & Coeficiente de Pearson \\
\hline H1.Atributo & 0,658 \\
H2.Precio y calidad & 0,435 \\
H3.Uso o aplicación & 0,649 \\
H4.Usuario del producto & 0,455 \\
H5.Clase de producto & 0,649 \\
H6.Competidor & 0,626 \\
H7.Emoción & 0,619 \\
\hline
\end{tabular}

Tabla 2. Coeficientes de correlación de Pearson para cada hipótesis.

Las 7 hipótesis de trabajo de las variables independientes se consideran probadas en función a que se trata de datos de opinión, con la observación de que las variables independientes: precio y calidad
$(0,435)$ y usuario del producto $(0,455)$ están en el límite inferior del rango de la tabla de significados de $r$ de 0,40 a 0,70 que significa correlación moderada, sustancial, aproximándose al rango de: $r$ de 0,20 a 0,40 que significa baja correlación, definida, pero baja. Adicionalmente, por medio del estudio de campo realizado en los 265 turistas nacionales e internacionales y visitantes locales, se permitió dar solución o respuesta al objetivo general planteado y con la finalidad de aportar resultados concretos avalados con la opinión y enfoques de los encuestados.

Por otra parte, en la discusión teórica y de acuerdo a los autores del modelo y del concepto de posicionamiento, que dieron soporte a la investigación teórica y práctica, se exponen los siguientes criterios:

Ries y Trout (1976) , considerados como los creadores del concepto de Posicionamiento en la década de los 70's, en su libro: "Posicionamiento: la batalla por su mente", al igual que Trout y Rivkin (1996) en su libro: "El nuevo posicionamiento", plantearon el problema sobre que las marcas, los productos y las líneas de productos se podían posicionar en una lista en la mente de los consumidores. Este planteamiento es aplicable y adecuado al problema de la investigación, y se considera que es el referente original y fundamental que se tiene en la mercadotecnia para hacer uso del posicionamiento en todo el mundo. 
Aunque existan otros autores clásicos como Kotler (1988), Stanton y Futrell (1989) y McCarthy (1987), que en sus libros de mercadotecnia hacen referencia y definen el concepto de posicionamiento, se puede apreciar que son basados en el concepto original de Ries y Trout (1989). Es importante considerar que en el modelo propuesto por Lamb et al., (2008) y utilizado en la presente investigación, se adapta al contexto para lograr el reposicionamiento del Barrio del Artista para aumentar su valor cultural artístico en Puebla, para efectos de comprobar el planteamiento y comprobación de la hipótesis.

En esta investigación se tomó como base el modelo de Lamb et al., (2008), ya que las 7 variables propuestas siguen siendo las bases para aplicar el modelo, y así poder adaptar este al contexto del reposicionamiento. Como resultado de la investigación se determinó que el comportamiento del modelo posicionamiento y sus 7 variables controlables (atributo, precio y calidad, uso o aplicación, usuario del producto, clase de producto, competidor y emoción) tienen un comportamiento directo al fundamentado en la teoría. Es importante mencionar que aún cuando se consideraron ediciones bibliográficas más recientes en los conceptos de posicionamiento, como es el caso del nuevo posicionamiento, no han cambiado en su esencia teórica.

\section{Conclusiones}

La presente investigación permitió conocer diferentes aspectos del posicionamiento a través de las 7 variables independientes: atributo, precio y calidad, uso o aplicación, usuario del producto, clase de producto, competidor y emoción, para aumentar el valor cultural artístico del Barrio del Artista en Puebla. Se observó que la temporada vacacional diciembre 2013 estuvo caracterizada por ser considerada una de las temporadas turísticas que repunta más altamente en la ciudad de Puebla.
Se observó que existen varios factores determinantes de posicionamiento para aumentar el valor cultural y artístico de El Barrio del Artista en Puebla. Estos pueden afectar en forma directa o indirecta a los turistas nacionales e internacionales así como a los visitantes locales para su visita a este espacio. No se encontró una teoría que conduzca a ordenar en forma jerárquica a los 7 factores. Dependerá del contexto donde se desarrollan. Tampoco existen modelos únicos para medir el reposicionamiento en el Barrio del Artista.

El estudio documental permitió analizar y sistematizar la información hemerobibliográfica sobre el tema de posicionamiento y reposicionamiento, que sirvió de base para la elaboración de un marco teórico acerca de los turistas y visitantes en el Barrio del Artista. El modelo de las 7 variables que se utilizó, en el cual se relacionan en forma sistémica a las 7 variables independientes seleccionadas con la variable dependiente (aumento del valor cultural artístico del Barrio del Artista en Puebla), consideró que todas las variables están interrelacionadas y que actúan en conjunto como un sistema, donde ninguna variable ejerce una influencia de forma independiente sobre el aumento del valor cultural artístico del Barrio del Artista en Puebla.

El objetivo general se logra con el modelo de posicionamiento de las 7 variables determinantes del Aumento del valor cultural artístico del Barrio del Artista en Puebla, que quedó definido en el diagrama de variables. El estudio de campo realizado fue con el objeto de analizar el comportamiento y la situación de cada factor. Los objetivos específicos se logran al describir el marco teórico y metodológico.

Se alcanzó el objetivo de la investigación porque se determinó que las variables independientes consideradas influyen en forma directamente proporcional y positiva en el aumento del valor cultural artístico del Barrio del Artista en Puebla; la hipótesis general y las siete hipótesis de trabajo que se sometieron a prueba dan evidencia a favor. 
Sin embargo, se debe tomar en cuenta que son juicios de valor y datos de opinión de los turistas nacionales e internacionales y que no dan evidencia a favor al $100 \%$. Aún cuando las hipótesis que se plantearon se observan lógicas, no existe información sistematizada acerca del tipo de intensidad de la asociación entre las variables y más en el sujeto de estudio considerado. Queda claro que la presente investigación tuvo como base un objeto de estudio específico, que a través de un método, llegó a resultados y conclusiones. De esta forma, al usar la teoría, la prueba y demostración para que se avance en el conocimiento sistematizado, permite seguir en el camino para el apoyo en la construcción del conocimiento científico.

\section{Referencias}

Acle, R., Villalobos, C., Santiesteban, N., Rodríguez, M., \& Zamora, M. (2014). Posicionamiento del Barrio del Artista en la ciudad de Puebla. En Carmona, G.N., Ramírez-Yuridia, G.S., \& Almanza-Serrano, L. (Ed.) Ciencias Administrativas y Sociales Handbook T-V: Congreso Interdisciplinario de Cuerpos Académicos (pp. 247-261). Madrid, España: ECORFAN.

Acle-Mena, R.S., \& Burguete-García, M.A. (2011). La influencia de la mezcla de mecadotecnia en el consumo por nostalgia. En XVI Congreso Internacional de Contaduría, Administración e Informática. Congreso llevado a cabo en la Universidad Nacional Autónoma de México, México D.F., México.

Acle-Mena, R.S., Domínguez-Y-López, J.E., \& Burguete-García, M.A. (2014). Posicionamiento y su relación en el consumo cultural artístico, caso estudio: Barrio el Artista en Puebla, México. En Lobo-Rodríguez, M.A. (Coordinadora), Congreso de Investigación Turística 2014. Congreso llevado a cabo en la Universidad Autónoma de Baja California, Tijuana, México.

Deana, A. (1986). Cosas de Puebla (Vol. Tomo III). Puebla, México: Talleres de Marco Antonio Fuentes Rodiles.

Fischer, L., \& Espejo, J. (2007). Mercadotecnia. México D.F., México: Mc Graw-Hill.
Gambini, B. (2010). Marketing: lo último en la batalla mental del posicionamiento al reposicionamiento. México D.F., México: Agro Enfoque.

Kotler, P. (1988). Marketing management:analysis, planning, implementation, and control. Nueva York, Estados Unidos: Prentice-Hall.

Kotler, P., \& Armstrong, G. (2001). Marketing. México D.F, México: Prentice Hall.

Lamb, C. (2011). Marketing (11a ed.). México D.F., México: Cengage Learning.

Lamb, C., Hair, J., \& Mc Daniel, C. (2008). Marketing. México D.F., México: Cenage Learning.

McCarthy, E.J. (1987). Manual of objective tests to accompany basic marketing: a managerial approach. Irwin, Estados Unidos: McGraw Hill.

Mármol-Sinclair, P., \& Ojeda-García, C.D. (2012). Marketing turístico (pp. 36-42). Madrid, España: Ediciones Paraninfo, SA.

Ries, A., \& Trout, J. (1976). Positioning: the battle for your mind. Nueva York, Estados Unidos: Warner Books Inc.

Stanton, W., Etzel, M., \& Walker, B. (2003). Fundamentos de Marketing. México D.F., México: Mc Graw-Hill.

Stanton W. J., \& Futrell, C. (1989). Fundamentos de Mercadeo. Nueva York, Estados Unidos: MacGraw-Hill.

Trout, J., \& Rivkin, S. (1996). El nuevo posicionamiento. México D.F., México: Mc Graw-Hill. 